\title{
6

\section{Evaluating the Revised American Society for Gastrointestinal Endoscopy Guidelines for Common Bile Duct Stone Diagnosis}

\author{
Jake S. Jacob', Michelle E. Lee', Erin Y. Chew ${ }^{2}$, Aaron P. Thrift ${ }^{3,4}$ and Robert J. Sealock ${ }^{1}$ \\ ${ }^{1}$ Section of Gastroenterology and Hepatology, Department of Medicine, Baylor College of Medicine, Houston, TX, ${ }^{2}$ Department of Internal \\ Medicine, Johns Hopkins Hospital, Baltimore, MD, ${ }^{3}$ Section of Epidemiology and Population Sciences, Department of Medicine, Baylor \\ College of Medicine, Houston, TX, ${ }^{4}$ Dan L Duncan Comprehensive Cancer Center, Baylor College of Medicine, Houston, TX, USA
}

Background/Aims: The American Society for Gastrointestinal Endoscopy (ASGE) revised its guidelines for risk stratification of patients with suspected choledocholithiasis. This study aimed to assess the diagnostic performance of the revision and to compare it to the previous guidelines.

Methods: We conducted a retrospective cohort study of 267 patients with suspected choledocholithiasis. We identified high-risk patients according to the original and revised guidelines and examined the diagnostic accuracy of both guidelines. We measured the association between individual criteria and choledocholithiasis.

Results: Under the original guidelines, 165 (62\%) patients met the criteria for high risk, of whom $79 \%$ had confirmed choledocholithiasis. The categorization had a sensitivity and specificity of $68 \%$ and $55 \%$, respectively, for the detection of choledocholithiasis. Under the revised guidelines, 86 (32\%) patients met the criteria for high risk, of whom $83 \%$ had choledocholithiasis. The revised categorization had a lower sensitivity and higher specificity of $37 \%$ and $80 \%$, respectively. The positive predictive value of the high-risk categorization increased with the revision, reflecting a potential decrease in diagnostic endoscopic retrograde cholangiopancreatograpies (ERCPs). Stone visualized on imaging had the greatest specificity for choledocholithiasis. Gallstone pancreatitis was not associated with the risk for choledocholithiasis.

Conclusions: The 2019 revision of the ASGE guidelines decreases the utilization of ERCP as a diagnostic modality and offers an improved risk stratification tool. Clin Endosc 2021;54:269-274

Key Words: Biliary tract disease; Choledocholithiasis; Endoscopic retrograde cholangiopancreatography; Endoscopic ultrasound; Magnetic resonance cholangiopancreatography

\section{INTRODUCTION}

Biliary gallstone disease is a common clinical problem worldwide. ${ }^{1,2}$ If left untreated, gallstone obstruction can lead to life-threatening illnesses, such as cholecystitis, pancreatitis, and/or cholangitis. Endoscopic retrograde cholangiopancreatography (ERCP) has been the principle procedure used in the

Received: April 15, 2020 Revised: July 10, 2020

Accepted: August 10, 2020

Correspondence: Jake S. Jacob

Section of Gastroenterology and Hepatology, Department of Medicine, Baylor College of Medicine, 1 Baylor Plaza, Houston, TX 77030, USA

Tel: +1-404-401-2688, Fax: +1-713-798-0951, E-mail: jake.jacob@bcm.edu ORCID: https://orcid.org/0000-0001-8180-3080

(cc) This is an Open Access article distributed under the terms of the Creative Commons Attribution Non-Commercial License (http://creativecommons.org/ licenses/by-nc/3.0) which permits unrestricted non-commercial use, distribution, and reproduction in any medium, provided the original work is properly cited. management of choledocholithiasis due to its diagnostic and therapeutic capabilities, ${ }^{3}$ and favorable risk-to-benefit profile in the appropriately applied patient. ${ }^{4}$ More recently, advanced imaging techniques, such as endoscopic ultrasound (EUS) and magnetic resonance cholangiopancreatography (MRCP), have been shown to offer a safer diagnostic alternative to ERCP. ${ }^{5}$ Ideally, patients are triaged using the least invasive diagnostic modality appropriate for their risk profiles.

To standardize the diagnosis and management of choledocholithiasis, the American Society for Gastrointestinal Endoscopy (ASGE) released a risk stratification tool in 2010 that categorized patients into low, intermediate, and high risk for choledocholithiasis, and offered management suggestions for each category. ${ }^{6}$ Several institutions have validated the risk stratification tool since $2010^{7-10}$ While the tool achieved its intended aim, it was found to inappropriately triage patients to 
higher risk profiles, leading to the overuse of diagnostic ERCPs (ERCP without intervention, due to the absence of choledocholithiasis) and patient exposure to potentially unnecessary complications. ${ }^{10}$ To address this concern, the ASGE recently modified its risk stratification tool to improve diagnostic accuracy and increase the use of less invasive imaging modalities, such as EUS and MRCP. ${ }^{11}$

We aimed to evaluate the original 2010 and revised 2019 guidelines for risk stratification of patients with suspected choledocholithiasis. We examined the diagnostic accuracy of both guidelines and assessed the performance of the individual criteria used in these guidelines.

\section{MATERIALS AND METHODS}

The Baylor College of Medicine Institutional Review Board approved this study. We reviewed the electronic medical records for all patients with suspected choledocholithiasis seen by the gastroenterology consult service at an urban county hospital between January 1, 2012, and December 31, 2017. The inclusion criteria comprised the presence of right upper quadrant and/or epigastric abdominal pain, abnormal liver function test results (LFTs), defined as twice the normal upper limit, or imaging suggestive of common bile duct (CBD) di- lation. Only patients who received confirmatory testing with ERCP or intraoperative cholangiogram (IOC) were included in the study. We excluded patients with clinical evidence of ascending cholangitis (due to the need for urgent biliary decompression), history of post-surgical gastric or biliary surgery (cholecystectomy, Roux-en-Y gastric bypass, Billroth I or II, choledochojejunostomy, or hepaticojejunostomy), prior ERCP, history of hepatobiliary malignancy, or incomplete data (defined as lack of confirmatory testing for choledocholithiasis).

For patients who met the study inclusion/exclusion criteria, two independent reviewers (JSJ and MEL) extracted data from their electronic medical records, including dates of admission and discharge, demographic characteristics, diagnostic lab test results at admission, $\mathrm{CBD}$ diameter, whether a stone was present on radiologic imaging, and final diagnosis. Demographic characteristics included age at admission, body mass index (BMI), gender, and race/ethnicity. Diagnostic lab tests included white blood cell count, total and direct bilirubin, alkaline phosphatase, aspartate aminotransferase, and alanine aminotransferase. Imaging modalities utilized included transabdominal US, computed tomography (CT) scan of the abdomen, EUS, MRCP, and IOC.

We retrospectively sub-classified patients into their respective ASGE risk category based on the risk stratification tools presented in both the 2010 (Supplementary Table 1) ${ }^{5}$ and 2019

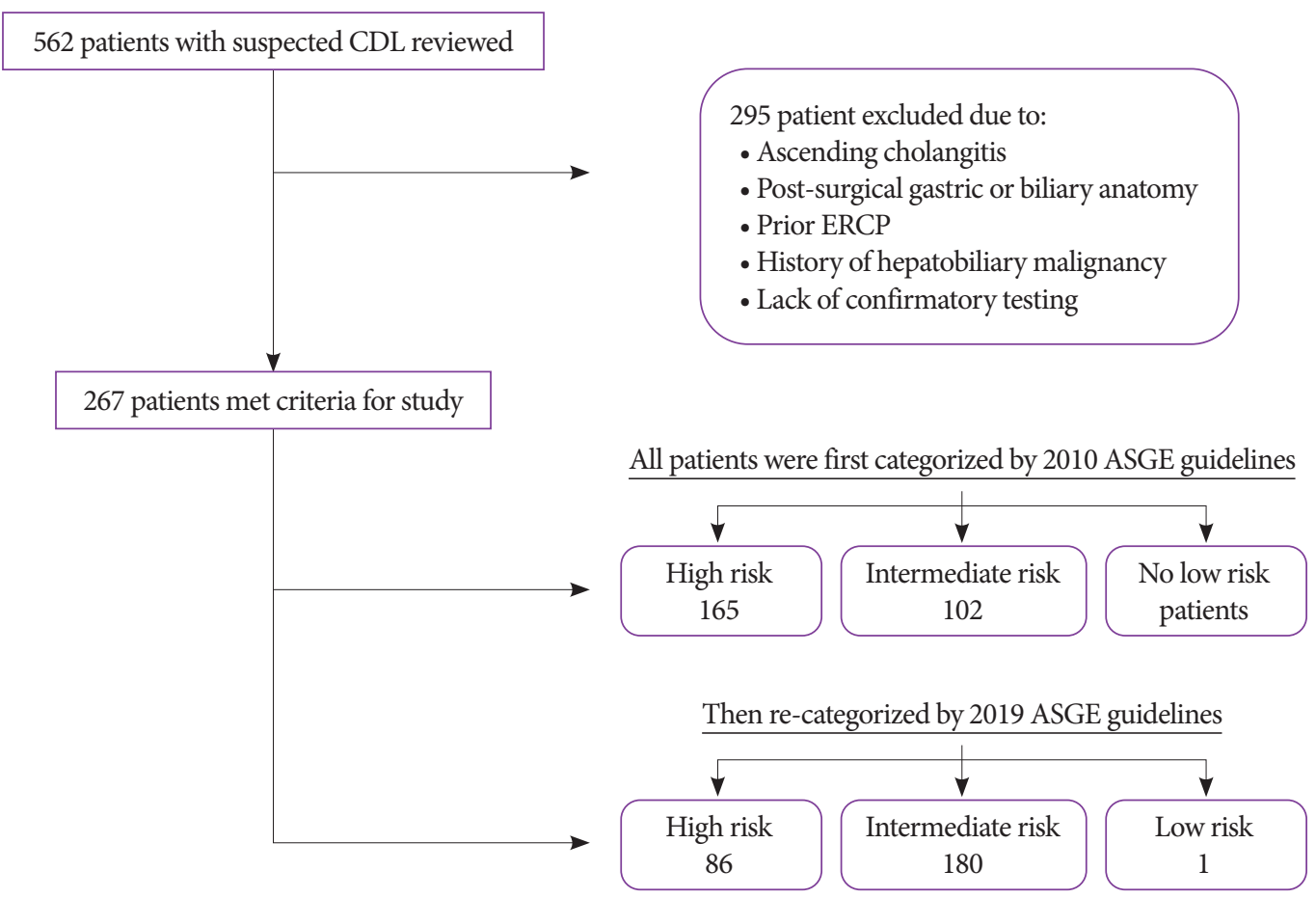

Fig. 1. Exclusion criteria and risk stratification of included patients with suspected choledocholithiasis (CDL). ASGE, American Society for Gastrointestinal Endoscopy; ERCP, endoscopic retrograde cholangiopancreatography. 
(Supplementary Table 2$)^{11}$ guidelines. Though not stated in the 2010 guidelines, we considered dilated CBD ( $>6 \mathrm{~mm}$ ) on CT scan as part of the strong criteria for risk of choledocholithiasis to maintain homogeneity with the 2019 guidelines. The gold standard confirmatory tests for choledocholithiasis were ERCP and IOC. Choledocholithiasis on ERCP was defined as the visualization of stone, stone fragments, or sludge in the duodenal lumen after ERCP. Choledocholithiasis on IOC was defined as the presence of a filling defect in the CBD or inadequate visualization of the lower end of the CBD due to intraductal calculus, as characterized by the performing surgeon (Fig. 1).

Our primary measures for this study included sensitivity, specificity, positive predictive value (PPV), and negative predictive value to determine and compare the diagnostic accuracies of the original 2010 and revised 2019 guidelines. In addition, we used logistic regression to estimate the odds ratios (ORs) and associated 95\% confidence intervals (CIs) for the associations between the individual risk stratification criteria and the final diagnosis of choledocholithiasis. The criteria for abnormal LFTs were excluded in this analysis because nearly all patients met these criteria. All statistical analyses were performed using Microsoft Excel and RStudio ver. 1.0.136.

\section{RESULTS}

\section{Patient characteristics}

A total of 267 patients met the inclusion/exclusion criteria for the study. The mean age at diagnosis was 40 years (standard deviation, 16), and the mean BMI was $30 \mathrm{~kg} / \mathrm{m}^{2}$ (standard deviation, 7.7). The majority of patients were female (78\%) and Hispanic (82\%). Among the 267 patients with suspected choledocholithiasis, 192 (72\%) had confirmed choledocholithiasis. The majority of patients had ERCP (98\%) as confirmatory testing compared to IOC (2\%) (Table 1$)$.

\section{Diagnostic accuracy of the ASGE guidelines}

Using the original (2010) criteria, 165 patients (62\%) were classified as high risk. Among these high-risk patients, 79\% had confirmed choledocholithiasis, while only $60 \%$ of the intermediate-risk group had confirmed choledocholithiasis. The high-risk categorization had a sensitivity of $68 \%$, specificity of 55\%, and PPV of 79\%. Under the revised guidelines (2019), 86 (32\%) patients were high risk. Among the high-risk and intermediate-risk patients under the new guidelines, $83 \%$ and $67 \%$ had confirmed choledocholithiasis, respectively. The 2019 high-risk categorization had a sensitivity of $37 \%$, specificity of $80 \%$, and PPV of $83 \%$ (Table 2).
Table 1. Baseline Characteristics of 267 Patients Included in the Study Analysis

\begin{tabular}{lcc}
\hline & $\boldsymbol{n}=\mathbf{2 6 7}$ & $\mathbf{( \% )}$ \\
\hline Age (average \pm SD), yr & $39.6 \pm 15.7$ & \\
Age $>55$ & 45 & 16.9 \\
\hline Gender, Female & 207 & 77.5 \\
BMI (average \pm SD) & $30.4 \pm 7.7$ & \\
$<18.5$ & 4 & 1.5 \\
18.5-24.9 & 57 & 21.3 \\
$25-29.9$ & 84 & 31.5 \\
$30-34.9$ & 66 & 24.7 \\
$35-39.9$ & 33 & 12.4 \\
$>40$ & 23 & 8.6 \\
Ethnicity & & \\
Hispanic & 218 & 81.6 \\
Non-hispanic white & 26 & 9.7 \\
African American & 14 & 5.2 \\
Asian & 5 & 1.9 \\
Choledocholithiasis & 192 & 72 \\
Final diagnostic testing (all risk categories) & & \\
ERCP & 262 & 98.1 \\
IOC & 5 & 1.87 \\
\hline BMI, body mass index; ERCP, endoscopic & & \\
\hline
\end{tabular}

BMI, body mass index; ERCP, endoscopic retrograde cholangiopancreatography; IOC, intraoperative cholangiogram; SD, standard deviation.

Table 2. Diagnostic Accuracy of 2010 and 2019 High Risk Stratification

\begin{tabular}{lcc}
\hline & $\mathbf{2 0 1 0}$ & $\mathbf{2 0 1 9}$ \\
\hline No. of HR patients (\%) & $165(62 \%)$ & $86(32 \%)$ \\
HR patients with CDL (\%) & $79 \%$ & $83 \%$ \\
OR (95\% CI) & $2.59(1.50-4.47)$ & $2.35(1.24-4.44)$ \\
Sensitivity & $68 \%(61-75)$ & $37 \%(30-44)$ \\
Specificity & $55 \%(43-66)$ & $80 \%(69-88)$ \\
PPV & $79 \%(75-83)$ & $83 \%(74-89)$ \\
NPV & $40 \%(33-47)$ & $33 \%(30-37)$ \\
\hline
\end{tabular}

CDL, choledocholithiasis; CI, confidence interval; HR, high risk; NPV, negative predictive value; OR, odds ratio; PPV, positive predictive value.

\section{Proportion of diagnostic ERCPs}

In our patient cohort, 34 of the 165 high-risk patients (21\%) underwent diagnostic ERCP when following the 2010 guidelines. If the 2019 guidelines were followed, 15 of the 86 high- 
Table 3. Association between Individual Criteria and Choledocholithiasis

\begin{tabular}{|c|c|c|c|c|c|}
\hline & $n$ & $\%$ with CDL & Sensitivity (95\% CI) & Specificity $(95 \%$ CI $)$ & OR $(95 \% \mathrm{CI})$ \\
\hline Stone on imaging & 39 & $85 \%$ & $17 \%(12-23)$ & $92 \%(83-97)$ & $2.39(0.96-5.96)$ \\
\hline CBD dilation & 92 & $83 \%$ & $40 \%(33-47)$ & $79 \%(68-87)$ & $2.42(1.29-4.51)$ \\
\hline Total bilirubin $>4$ & 164 & $77 \%$ & $66 \%(59-73)$ & $51 \%(39-62)$ & $2.01(1.17-3.45)$ \\
\hline $1.8<$ Total bilirubin $<4$ & 127 & $73 \%$ & $48 \%(41-56)$ & $55 \%(43-66)$ & $1.13(0.66-1.94)$ \\
\hline Age $>55$ & 45 & $71 \%$ & $17 \%(12-23)$ & $83 \%(72-90)$ & $0.95(0.47-1.94)$ \\
\hline Gallstone pancreatitis & 74 & $53 \%$ & $20 \%(15-27)$ & $53 \%(41-65)$ & $0.29(0.16-0.52)$ \\
\hline
\end{tabular}

$\mathrm{CBD}$, common bile duct; $\mathrm{CDL}$, choledocholithiasis; CI, confidence interval; OR, odds ratio.

risk patients (17.4\%) would have received a diagnostic ERCP. The difference in the rates of diagnostic ERCP did not meet statistical significance $(p=0.548)$.

\section{Association between individual criteria and final diagnosis of choledocholithiasis}

Among patients with a stone visualized on imaging, $85 \%$ had confirmed choledocholithiasis. Moreover, patients with a stone visualized on imaging had a 2.4-fold higher risk of choledocholithiasis than those without (OR, 2.39; 95\% CI, 0.96-5.96) (Table 3). Other criteria associated with the increased risk for choledocholithiasis included CBD dilation (OR, 2.42; 95\% CI, 1.29-4.51) and serum bilirubin $>4 \mathrm{mg} / \mathrm{dL}$ (OR, 2.01; 95\% CI, 1.17-3.45). The criteria for serum bilirubin between 1.8 and $4 \mathrm{mg} / \mathrm{dL}$ (OR, 1.13; 95\% CI, 0.66-1.94) and age $>55$ (OR, $0.95 ; 95 \%$ CI, 0.47-1.94) did not meet statistical significance in predicting choledocholithiasis. The diagnosis of gallstone pancreatitis was not associated with the increased risk of choledocholithiasis (Table 3).

\section{DISCUSSION}

In 2010, the ASGE released a risk stratification tool using a weighted combination of clinical predictors to categorize patients into three tiers of risk for choledocholithiasis (low: $<10 \%$, intermediate: $10 \%-50 \%$, high: $>50 \%$ ) and provide management recommendations based on risk profiles. ${ }^{5}$ The tool was created with data from previous studies examining the prognostic utility of individual clinical criteria, ${ }^{12-14}$ though it had not been validated at the time of publication. The guidelines aimed to maximize the efficient utilization of the various diagnostic and therapeutic modalities for choledocholithiasis, including EUS, MRCP, IOC, ERCP, and laparoscopic cholecystectomy. In general, low-risk patients were managed with elective therapeutic intervention, intermediate-risk patients with further diagnostic evaluation of the biliary tree, and highrisk patients with additional evaluation with a modality that allowed therapeutic intervention if needed.

Since 2010, multiple institutions have assessed the diagnostic accuracy and clinical utility of the original ASGE risk stratification tool. ${ }^{6-9}$ In all of these studies, between $10 \%$ and $50 \%$ of the patients in the intermediate-risk group and $>50 \%$ of those in the high-risk group had confirmed choledocholithiasis, as intended. In studies including patients with clinical evidence of cholangitis, the high-risk classification had sensitivities ranging from $68 \%$ to $80 \%$ and specificities ranging from $44 \%$ to $74 \% .{ }^{6,79}$ In a study excluding patients with cholangitis, the sensitivity and specificity was $47 \%$ and $73 \%$, respectively, for the high-risk classification. ${ }^{8}$ We recommend excluding patients with signs of cholangitis when evaluating the diagnostic accuracy of the tool, as these patients require urgent biliary decompression irrespective of the presence of choledocholithiasis.

In our cohort of 267 included patients, $79 \%$ of the high-risk patients had confirmed choledocholithiasis, in accordance with the intended rate of $>50 \%$. In the intermediate-risk group, excluding the patients who did not have gold standard diagnostic testing prevented accurate assessment of the risk profile. The measured sensitivity (68\%) and specificity (55\%) of the high-risk category aligned with the measurements conducted at other institutions. ${ }^{7-10}$

A major concern regarding the 2010 risk stratification tool was the large number of diagnostic ERCPs performed, suggesting that the high-risk categorization was overly sensitive. These patients were exposed to unnecessary radiation and potential complications, including bleeding, perforations, and post-ERCP pancreatitis. ${ }^{3}$ The PPV of the high-risk category is a key diagnostic measure that is inversely proportional to the rate of diagnostic ERCPs. The studies previously cited reported PPVs of the high-risk classification ranging from 56\% to $87 \%$. In our cohort, the high-risk classification had a measured 
PPV of 79\%, similar to previous studies. Of the 165 high-risk patients in our study, 34 (21\%) underwent diagnostic ERCP as per the 2010 management algorithm.

In 2019, the risk stratification tool was revised to address the concern surrounding the relatively large number of diagnostic ERCPs performed. The goal was to reduce the number of diagnostic ERCPs by increasing utilization of EUS and MRCP, which are safer and have similar diagnostic performances. ${ }^{15,16}$

In order to achieve these goals, the criteria for the high-risk category were modified between 2010 and 2019. The clinical predictors were simplified into two groups: high risk and moderate risk. In the high-risk group, evidence of cholangitis remained the same, while stone visualized on imaging was expanded to include CT imaging. The third criterion was made stricter by requiring the presence of serum bilirubin $>4 \mathrm{mg} /$ $\mathrm{dL}$ in combination with a dilated CBD on imaging. Serum bilirubin between 1.8 and $4 \mathrm{mg} / \mathrm{dL}$ was changed to moderate risk. Finally, the diagnosis of gallstone pancreatitis was removed owing to the lack of predictive value. .17,18 $^{8}$

As intended, when applied to our cohort, fewer patients met the high-risk criteria (86 vs. 165 as per the prior guidelines). The PPV of the new high-risk classification improved to $83 \%$, which would have led to a smaller percentage (17\%) of diagnostic ERCPs, though this difference did not meet statistical significance in our cohort. The intermediate-risk group expanded from 102 to 181 patients, thereby increasing the pool of patients who would undergo further biliary imaging before intervention with a therapeutic modality. Only one patient moved from intermediate risk to low risk based on the removal of gallstone pancreatitis as a moderate risk predictor. Due to reasons previously mentioned, the risk profile of the intermediate- and low-risk groups could not be accurately measured.

We found that the visualization of a CBD stone on imaging was the most specific risk factor for choledocholithiasis, followed by serum bilirubin $>4 \mathrm{mg} / \mathrm{dL}$ and CBD dilation. All three of these criteria were highly specific and with poor sensitivities. The diagnosis of gallstone pancreatitis was not associated with the diagnosis of choledocholithiasis. The only patient in our study who moved from intermediate risk to low risk with the removal of the gallstone pancreatitis criterion did not have choledocholithiasis on confirmatory testing. Previous studies have also found that the majority of patients with gallstone pancreatitis do not have evidence of choledocholithiasis on cholangiogram or $\mathrm{ERCP}^{19,20}$ and in our cohort, gallstone pancreatitis was not associated with choledocholithiasis.

A limitation of this study was its retrospective nature, which prevented the evaluation of diagnostic accuracy of these guidelines in the intermediate-risk group. Under the current guidelines, intermediate-risk patients without evidence of

choledocholithiasis on EUS or MRCP often do not undergo confirmatory testing. Since EUS and MRCP do not have perfect negative predictive values, it is challenging to know the true prevalence of choledocholithiasis in this intermediate-risk group. We did not measure the cost-effectiveness of the different guidelines; as per the 2019 guidelines, several patients would be triaged with EUS or MRCP prior to definitive testing, which can increase costs. Finally, the patient population of this study was predominantly Hispanic and female, which may limit the generalizability of results compared with other patient populations.

In conclusion, the guidelines for risk stratification and management of suspected choledocholithiasis were revised to increase the utilization of EUS and MRCP and reduce the number of diagnostic ERCPs. We performed a retrospective comparison of the original and revised guidelines and confirmed that the update achieved its goals. The results from analysis of individual criteria supported the decision to remove the gallstone pancreatitis as a criterion in risk stratification.

The next step in improving the management of choledocholithiasis is to evaluate and optimize cost-effectiveness of biliary imaging. While EUS and MRCP offer a safer approach for patients, different strategies, such as tandem EUS and ERCP, can be used to improve the diagnostic accuracy and reduce costs associated with these diagnostic modalities. ${ }^{21}$

Conflicts of Interest

The authors have no potential conflicts of interest.

Funding

None.

Author Contributions

Conceptualization: Jake S. Jacob, Erin Y. Chew, Robert J. Sealock

Data curation: ISJ, Michelle E. Lee, EYC

Formal analysis: JSJ, Aaron P. Thrift

Funding acquisition: RJS

Investigation: EYC, RJS

Methodology: EYC, RJS

Project administration: JSJ, RJS

Resources: RJS

Software: JSJ, APT

Supervision: JSJ, RJS

Validation: JSJ, APT, RJS

Visualization: JSJ, APT

Writing-original draft: JSJ

Writing-review\&editing: JSJ, MEL, EYC, APT, RJS

ORCID

Jake S. Jacob:

Michelle E. Lee:

Erin Y. Chew:

Aaron P. Thrift:

Robert J. Sealock: https://orcid.org/0000-0001-8180-3080 https://orcid.org/0000-0003-0288-6362 https://orcid.org/0000-0001-6061-0706 https://orcid.org/0000-0002-0084-5308 https://orcid.org/0000-0003-0963-9727 


\section{REFERENCES}

1. Shaffer EA. Gallstone disease: epidemiology of gallbladder stone disease. Best Pract Res Clin Gastroenterol 2006;20:981-996.

2. Savides TJ. EUS-guided ERCP for patients with intermediate probability for choledocholithiasis: is it time for all of us to start doing this? Gastrointest Endosc 2008;67:669-672

3. Andriulli A, Loperfido S, Napolitano G, et al. Incidence rates of post-ERCP complications: a systematic survey of prospective studies. Am J Gastroenterol 2007;102:1781-1788.

4. Tse F, Barkun JS, Romagnuolo J, Friedman G, Bornstein JD, Barkun AN Nonoperative imaging techniques in suspected biliary tract obstruction. HPB (Oxford) 2006;8:409-425.

5. ASGE Standards of Practice Committee; Maple JT, Ben-Menachem T, et al. The role of endoscopy in the evaluation of suspected choledocholithiasis. Gastrointest Endosc 2010;71:1-9.

6. Kuzu UB, Ödemiş B, Dişibeyaz S, et al. Management of suspected common bile duct stone: diagnostic yield of current guidelines. HPB (Oxford) 2017;19:126-132.

7. He H, Tan C, Wu J, et al. Accuracy of ASGE high-risk criteria in evaluation of patients with suspected common bile duct stones. Gastrointest Endosc 2017;86:525-532

8. Adams MA, Hosmer AE, Wamsteker EJ, et al. Predicting the likelihood of a persistent bile duct stone in patients with suspected choledocholithiasis: accuracy of existing guidelines and the impact of laboratory trends. Gastrointest Endosc 2015;82:88-93.

9. Sethi S, Wang F, Korson AS, et al. Prospective assessment of consensus criteria for evaluation of patients with suspected choledocholithiasis. Dig Endosc 2016;28:75-82.

10. Costi R, Gnocchi A, Di Mario F, Sarli L. Diagnosis and management of choledocholithiasis in the golden age of imaging, endoscopy and laparoscopy. World J Gastroenterol 2014;20:13382-13401.

11. ASGE Standards of Practice Committee; Buxbaum JL, Abbas Fehmi SM, et al. ASGE guideline on the role of endoscopy in the evaluation and management of choledocholithiasis. Gastrointest Endosc 2019;89:10751105.e15.
12. Abboud PA, Malet PF, Berlin JA, et al. Predictors of common bile duct stones prior to cholecystectomy: a meta-analysis. Gastrointest Endosc 1996;44:450-455.

13. Tse F, Barkun JS, Barkun AN. The elective evaluation of patients with suspected choledocholithiasis undergoing laparoscopic cholecystectomy. Gastrointest Endosc 2004;60:437-448.

14. Pezzoli A, Morsiani E, Capprellari L, et al. Predicting the presence of choledocholithiasis in patients with symptomatic cholelitihasis. Dig Liver Dis 2001;33(Suppl 1):A20.

15. Tse F, Liu L, Barkun AN, Armstrong D, Moayyedi P. EUS: a meta-analysis of test performance in suspected choledocholithiasis. Gastrointest Endosc 2008;67:235-244.

16. Romagnuolo J, Bardou M, Rahme E, Joseph L, Reinhold C, Barkun AN. Magnetic resonance cholangiopancreatography: a meta-analysis of test performance in suspected biliary disease. Ann Intern Med 2003;139:547557.

17. Rubin MI, Thosani NC, Tanikella R, Wolf DS, Fallon MB, Lukens FJ. Endoscopic retrograde cholangiopancreatography for suspected choledocholithiasis: testing the current guidelines. Dig Liver Dis 2013;45:744749 .

18. Scheiman JM, Carlos RC, Barnett JL, et al. Can endoscopic ultrasound or magnetic resonance cholangiopancreatography replace ERCP in patients with suspected biliary disease? A prospective trial and cost analysis. Am J Gastroenterol 2001;96:2900-2904.

19. Chang L, Lo SK, Stabile BE, Lewis RJ, de Virgilio C. Gallstone pancreatitis: a prospective study on the incidence of cholangitis and clinical predictors of retained common bile duct stones. Am J Gastroenterol 1998;93:527-531.

20. Cohen ME, Slezak L, Wells CK, Andersen DK, Topazian M. Prediction of bile duct stones and complications in gallstone pancreatitis using early laboratory trends. Am J Gastroenterol 2001;96:3305-3311.

21. Chu YL, Wang XF, Gao XZ, et al. Endoscopic ultrasonography in tandem with endoscopic retrograde cholangiopancreatography in the management of suspected distal obstructive jaundice. Eur J Gastroenterol Hepatol 2013;25:455-459. 